\title{
Numerical Solution of Partial Differential Equations with Fractional Variable Coefficients Using New Iterative Method (NIM)
}

\author{
Falade K. $\mathrm{I}^{1^{*}}$, Tiamiyu A.T ${ }^{2}$ \\ ${ }^{1}$ Department of Mathematics, Faculty of Computing and Mathematical Sciences, Kano University of Science \\ and Technology, P.M.B 3244 Wudil Kano State, Nigeria \\ ${ }^{2}$ Department of Mathematics, Federal University of Technology, Minna Niger State, Nigeria
}

Received: 24 March 2020; Accepted: 03 May 2020; Published: 08 June 2020

\begin{abstract}
In this paper, we studied to obtain numerical solutions of partial differential equations with fractional variable coefficient by MAPLE 18 software algorithm on New Iterative Method. We examined and investigated behaviours of the fractional variable coefficients (Even and Odd) on first order partial differential equation; we obtain numerical solution and plot 2D/3D graphs representation of eight (8) cases for the study of the sequential trend of the fractional coefficients. The simplicity and the accuracy of the proposed numerical scheme are verified. More numerical examples will be used in the future for further testing the ability of the proposed scheme for solving some classical problems in engineering sciences.
\end{abstract}

Index Terms: Partial differential equation PDE, MAPLE 18 mathematical software, new iterative algorithm, fractional variable coefficients (Even and Odd).

(C) 2020 Published by MECS Publisher. Selection and/or peer review under responsibility of the Research Association of Modern Education and Computer Science

\section{Introduction}

Partial differential equations (PDEs) arise in all fields of engineering and sciences. Most real physical processes are governed by partial differential equations. In many cases, simplifying approximations are made to reduce the governing PDEs to ordinary differential equations (ODEs) or even to algebraic equations. However, because of the ever increasing requirement for more accurate modelling of physical processes, engineers and scientists are more and more required to solve the actual PDEs that govern the physical problem being examined and investigated. A partial differential equation (PDE) is an equation stating a relationship between function of two or more independent variables and the partial derivatives of this function with respect to these independent variables. Partial differential equations with variable coefficient have arisen from daily activities

*Corresponding author.

E-mail Address: faladekazeem2016@kustwudil.edu.ng 
such as physic, chemistry, applied sciences, technology of space, communication systems, nonlinear science, numerical simulation and control engineering problems. The coefficients in a partial differential equation depend on the structure of physical problem [1,2]. Computational and numerical techniques are very important in understanding the physical phenomena because of the longstanding challenges faced in analytical solution of some classical partial differential equation [3]. In recent years, various numerical and analytical methods have been developed and successfully used to solve PDEs such as the differential transform method (DTM) [4], the homotopy analysis method (HAM) [5], the Adomian decomposition method (ADM) [6], the variational iteration method (VIM) [7], the homotopy perturbation method (HPM) [8], the Laplace decomposition method (LDM) [9], the Sumudu decomposition method (SDM) [10] and [11] proposed the discrete Adomian decomposition method for the solution of integer order Schrodinger equation. Some of these methods use specific transformations and others give the solution as a series which converges to the exact solution.

Solving and writing reliable algorithms for the numerical solution for Partial Differential Equations can be therefore a quite difficult task for users with no particular expertise in computational mathematics. Thus the objectives of this paper is to illustrate and write the basic codes of MAPLE 18 mathematical software using New Iterative procedures for the numerical solution of fractional variable coefficient of the first order partial differential equation in order to overcome the mathematical stress of integral involves.

In this paper, we shall investigate effect of fractional variable coefficient on first order partial differential equation of the form:

$$
\frac{\partial u(x, t)}{\partial t}+\lambda(x) \frac{\partial u(x, t)}{\partial x}+u(x, t)=g(x) \quad 0<x<1, t>0
$$

subject to initial condition

$$
u(x, t)=f(x)
$$

Where $\lambda(x)=\frac{x}{k}$ such that $k \in \mathbb{R}^{+}, g(x)$ is a known function and $f(x)$ is a polynomial function.

In solving PDE problems like that of (1), most of these methods come with their drawbacks of huge computational work, but the NIM as one of the newly introduced method by Daftardar-Gejji and Jafari is user friendly, simple to understand and easier to implement using computer packages $[12,13,14,15]$.

This paper is concern with usage of MAPLE 18 software algorithm by New Iterative Method to examine the numerical trend of fractional variable coefficient of partial differential equation (1) couple with initial condition (2). The structure of the rest of the paper is as follows: In section II presents the New Iterative Method (NIM) and the algorithm for solving the equation in (1), section III presents the numerical experiment for eight cases (8) of using the NIM to solve the equation (1) with initial conditions, results, 2D and 3D graphs are presented in section IV. Finally, relevant discussion and conclusions are drawn in section V.

\section{The Description of The New Iterative Method (NIM)}

In this section, we present the New Iterative Method (NIM) as a numerical technique for solving functional equations. Consider a non-linear functional equation of the form:

$$
u(\bar{x})=f(\bar{x})+N(u((\bar{x})))
$$

where $\mathrm{N}$ a nonlinear operator from a Banach is space $B \rightarrow B$ and $f(\bar{x})$ is a known function and $\bar{x}=\left(x_{1}, x_{2}, x_{3}, \ldots x_{n}\right)$. We need to obtain the solution $u(\bar{x})$ of (3) having the series form;

$$
u(\bar{x})=\sum_{j=0}^{\infty} u_{j}(\bar{x})
$$


The nonlinear operator which is on the right-hand side of (3) can be decomposed as follow:

$N\left(\sum_{j=0}^{\infty} u_{j}(\bar{x})\right)=N\left(u_{0}\right)+\sum_{i=1}^{\infty}\left\{N\left(\sum_{j=0}^{i} u_{j}\right)-N\left(\sum_{j=0}^{i-1} u_{j}\right)\right\}$

Substituting equations (4) and (5) into the equation (3); becomes;

$\sum_{i=0}^{\infty} u_{i}(\bar{x})=f(\bar{x})+N\left(u_{0}\right)+\sum_{i=1}^{\infty}\left\{N\left(\sum_{j=0}^{i} u_{j}\right)-N\left(\sum_{j=0}^{i-1} u_{j}\right)\right\}$

The recurrence relation is given by;

$$
\left\{\begin{array}{c}
u_{0}=f \\
u_{1}=N\left(u_{0}\right) \\
\vdots \\
\vdots \\
u_{m+1}=N\left(u_{0}+u_{1}+\cdots+u_{m}\right)-N\left(u_{0}+u_{1}+\cdots+u_{m-1}\right) \\
m=1,2,3, . .
\end{array}\right.
$$

then.

$N\left(u_{0}+u_{1}+\cdots+u_{m}\right)=N\left(u_{0}+u_{1}+\cdots+u_{m-1}\right) \quad m=1,2,3 \ldots$

and

$\sum_{i=0}^{\infty} u_{i}=f+N\left(\sum_{i=0}^{\infty} u_{i}\right)$

The $k-$ term approximate solution of (3) is given by;

$u=u_{0}+u_{1}+\cdots+u_{k-1}$

\section{NIM Algorithm for fractional variable coefficient $\lambda(x)$ PDE}

We consider equation (1)

$\frac{\partial u(x, t)}{\partial t}+\lambda(x) \frac{\partial u(x, t)}{\partial x}+u(x, t)=g(x)$

We further express as;

$\frac{\partial u(x, t)}{\partial t}=g(x)-\lambda(x) \frac{\partial u(x, t)}{\partial x}-u(x, t)$

Couple with initial condition

$u(x, 0)=f(x)$

Where $\frac{\partial u(x, y)}{\partial t}$ is a linear function and $g(x)-\lambda(x) \frac{\partial u(x, t)}{\partial x}-u(x, t)$ is an analytical function of $\mathrm{u}$ and $\partial u(x, y)$ (partial derivatives of $u(x, t)$ with respect to $\mathrm{x}$ and $\mathrm{t}$ ) with the initial value problem. (12) to (13) is equivalent to the following integral equation: 
$u(x, t)-u(x, 0)=\int_{0}^{t}\left(g(x)-\lambda(x) \frac{\partial u(x, t)}{\partial x}-u(x, t)\right) d t$

$u(x, t)=u(x, 0) \int_{0}^{t}\left(g(x)-\lambda(x) \frac{\partial u(x, t)}{\partial x}-u(x, t)\right) d t$

$u(x, t)=f(x)+\int_{0}^{t}\left(g(x)-\lambda(x) \frac{\partial u(x, t)}{\partial x}-u(x, t)\right) d t$

$u(\bar{x})=f(x)+N(u(\bar{x}))$

Where $f(x)$ is the known function and $N(u)=\int_{0}^{t}\left(g(x)-\lambda(x) \frac{\partial u(x, t)}{\partial x}-u(x, t)\right) d t$. We get the solution of (17) following the procedure in (7).

\section{Using MAPLE 18 mathematical software Algorithm for NIM as follows:}

$$
\left\{\begin{array}{c}
\text { restart: } \\
\lambda(x)=\frac{x}{k} \\
f(x):=A \\
g(x):=B \\
u_{0}:=f(x)+t * g(x) \\
N:=C ; \\
\text { for } i \text { from } 0 \text { to } N \text { do } \\
e:=\lambda * \operatorname{diff}\left(u_{i}, x\right)+u_{i} ; \\
f:=-\operatorname{int}(e,[t=0 \ldots t]) \\
u_{i+1}:=\text { value }(f) \\
\text { end do } \\
U:=\operatorname{sum}\left(u_{j}, j=0,1 \ldots N+1\right)
\end{array}\right.
$$

where $\mathrm{C}$ is the iteration step length.

\section{Computational Experiment}

In this section, we examine the trend movement of $\lambda(x)$ using NIM MAPLE 18 algorithm developed in (8). The examples considered here are first order partial differential equation with fractional variable coefficient and quadratic function $g(x)$. We have successfully solved the PDE with specific initial condition as follow:

$\frac{\partial u(x, t)}{\partial t}+\lambda(x) \frac{\partial u(x, t)}{\partial x}+u(x, t)=g(x) \quad 0<x<1, t>0$

subject to initial condition

$u(x, t)=f(x)$

where

$$
\lambda(x)=\left\{\begin{array}{l}
\frac{x}{k} ; k=1,3,5,7 \ldots(\text { odd }) \\
\frac{x}{k} ; k=2,4,6,8, \ldots(\text { even })
\end{array},\left\{\begin{array}{l}
f(x)=A \\
g(x)=B
\end{array}\right.\right.
$$


Substitute the above given functions into equation (8) on each cases 1 to 8 , we obtain the following approximate solutions $u(x, t)$ presented in Tables 1 to 4 .

Table 1. Numerical solution $u(x, t)$ for case 1 and case 2

\begin{tabular}{|l|l|l|l|l|l|}
\hline \multicolumn{2}{|c|}{$u(x, t)$} & \multicolumn{1}{|c|}{$\lambda(x)=\frac{x}{1}, A=2 x^{4}, B=3 x, C=75$} & \multicolumn{2}{c|}{$\lambda(x)=\frac{x}{2}, A=2 x^{4}, B=3 x, C=75$} \\
\hline$x$ & $t$ & Exact Solution & NIM Solution & Exact Solution & NIM Solution \\
\hline 0 & 0 & 0.0000000000 & 0.0000000000 & 0.0000000000 & 0.0000000000 \\
\hline 0.1 & 0.1 & 0.0273116931 & 0.0273116932 & 0.0280065683 & 0.0280065684 \\
\hline 0.2 & 0.2 & 0.1000812004 & 0.1000812004 & 0.1054289090 & 0.1054289089 \\
\hline 0.3 & 0.3 & 0.2066494724 & 0.2066494724 & 0.2240095375 & 0.2240095375 \\
\hline 0.4 & 0.4 & 0.3373317881 & 0.3373317879 & 0.3763718348 & 0.3763718348 \\
\hline 0.5 & 0.5 & 0.4843510439 & 0.4843510442 & 0.5555247172 & 0.5555247175 \\
\hline 0.6 & 0.6 & 0.6418300175 & 0.6418300178 & 0.7549618802 & 0.7549618796 \\
\hline 0.7 & 0.7 & 0.8055739720 & 0.8055739729 & 0.9688907281 & 0.9688907284 \\
\hline 0.8 & 0.8 & 0.9727283494 & 0.9727283443 & 1.1924054080 & 1.1924054080 \\
\hline 0.9 & 0.9 & 1.1414237260 & 1.1414237250 & 1.4215546050 & 1.4215546050 \\
\hline 1.0 & 1.0 & 1.3104729690 & 1.3104729820 & 1.6533138160 & 1.6533138130 \\
\hline
\end{tabular}

Table 2. Numerical solution $u(x, t)$ for case 3 and case 4

\begin{tabular}{|l|l|l|l|l|l|}
\hline \multicolumn{2}{|c|}{$u(x, t)$} & \multicolumn{2}{|c|}{$\lambda(x)=\frac{x}{3}, A=2 x^{4}, B=3 x, C=75$} & \multicolumn{2}{c|}{$\lambda(x)=\frac{x}{4}, A=2 x^{4}, B=3 x, C=75$} \\
\hline$x$ & $t$ & Exact Solution & NIM Solution & Exact Solution & NIM Solution \\
\hline 0 & 0 & 0.0000000000 & 0.0000000000 & 0.0000000000 & 0.0000000000 \\
\hline 0.1 & 0.1 & 0.0282443811 & 0.0282443811 & 0.0283644896 & 0.0283644853 \\
\hline 0.2 & 0.2 & 0.1073389328 & 0.1073389328 & 0.1083206483 & 0.1083206483 \\
\hline 0.3 & 0.3 & 0.2305786509 & 0.2305786509 & 0.2340424679 & 0.2340424678 \\
\hline 0.4 & 0.4 & 0.3921523273 & 0.3921523275 & 0.4007362096 & 0.4007362096 \\
\hline 0.5 & 0.5 & 0.5863311439 & 0.5863311441 & 0.6036712159 & 0.6036712161 \\
\hline 0.6 & 0.6 & 0.8073238314 & 0.8073238315 & 0.8378617042 & 0.8378617038 \\
\hline 0.7 & 0.7 & 1.0494181500 & 1.0494181490 & 1.0980876690 & 1.0980876690 \\
\hline 0.8 & 0.8 & 1.3072028490 & 1.3072028520 & 1.3790651010 & 1.3790651010 \\
\hline 0.9 & 0.9 & 1.5757690460 & 1.5757690450 & 1.6756558710 & 1.6756558710 \\
\hline 1.0 & 1.0 & 1.8508503750 & 1.8508503770 & 1.9830590530 & 1.9830590520 \\
\hline
\end{tabular}


Table 3. Numerical solution $u(x, t)$ for case 5 and case 6

\begin{tabular}{|l|l|l|l|l|l|}
\hline \multicolumn{2}{|c|}{$u(x, t)$} & \multicolumn{2}{|c|}{$\lambda(x)=\frac{x}{5}, A=2 x^{4}, B=3 x, C=75$} & \multicolumn{2}{c|}{$\lambda(x)=\frac{x}{6}, A=2 x^{4}, B=3 x, C=75$} \\
\hline$x$ & $t$ & Exact Solution & NIM Solution & Exact Solution & NIM Solution \\
\hline 0 & 0 & 0.0000000000 & 0.0000000000 & 0.0000000000 & 0.0000000000 \\
\hline 0.1 & 0.1 & 0.0284369448 & 0.0284369449 & 0.0284854123 & 0.0284854141 \\
\hline 0.2 & 0.2 & 0.1089186337 & 0.1089186337 & 0.1093211231 & 0.1093211231 \\
\hline 0.3 & 0.3 & 0.2361832772 & 0.2361832771 & 0.2376378418 & 0.2376378418 \\
\hline 0.4 & 0.4 & 0.4061383242 & 0.4061383236 & 0.4098524679 & 0.4098524689 \\
\hline 0.5 & 0.5 & 0.6148066620 & 0.6148066623 & 0.6225653035 & 0.6225653038 \\
\hline 0.6 & 0.6 & 0.8578947760 & 0.8578947764 & 0.8720513113 & 0.8720513124 \\
\hline 0.7 & 0.7 & 1.1307172480 & 1.1307172480 & 1.1541118510 & 1.1541118520 \\
\hline 0.8 & 0.8 & 1.4283054480 & 1.4283054490 & 1.4641292920 & 1.4641292930 \\
\hline 0.9 & 0.9 & 1.7455927400 & 1.7455927400 & 1.7972211760 & 1.7972211780 \\
\hline 1.0 & 1.0 & 2.0776122450 & 2.0776122460 & 2.1484286300 & 2.1484286310 \\
\hline
\end{tabular}

Table 4. Numerical solution $u(x, t)$ for case 7 and case 8

\begin{tabular}{|l|l|l|l|l|l|}
\hline \multicolumn{2}{|c|}{$u(x, t)$} & \multicolumn{2}{|c|}{$\lambda(x)=\frac{x}{7}, A=2 x^{4}, B=3 x, C=75$} & \multicolumn{2}{c|}{$\lambda(x)=\frac{x}{8}, A=2 x^{4}, B=3 x, C=75$} \\
\hline$x$ & $t$ & Exact Solution & NIM Solution & Exact Solution & NIM Solution \\
\hline 0 & 0 & 0.0000000000 & 0.0000000000 & 0.0000000000 & 0.0000000000 \\
\hline 0.1 & 0.1 & 0.0285201128 & 0.0285201128 & 0.0285461824 & 0.0285461824 \\
\hline 0.2 & 0.2 & 0.1096105258 & 0.1096105257 & 0.1098286348 & 0.1098286350 \\
\hline 0.3 & 0.3 & 0.2386906511 & 0.2386906512 & 0.2394879964 & 0.2394879963 \\
\hline 0.4 & 0.4 & 0.6282817169 & 0.6282817164 & 0.4146291280 & 0.4146291273 \\
\hline 0.5 & 0.5 & 0.6282817169 & 0.6282817174 & 0.6326687198 & 0.6326687194 \\
\hline 0.6 & 0.6 & 0.8825866475 & 0.8825866477 & 0.8907325828 & 0.8907325830 \\
\hline 0.7 & 0.7 & 1.1717027220 & 1.1717027220 & 1.1854092310 & 1.1854092330 \\
\hline 0.8 & 0.8 & 1.4913483360 & 1.4913483360 & 1.5127230240 & 1.5127230260 \\
\hline 0.9 & 0.9 & 1.8368600590 & 1.8368600580 & 1.8682321030 & 1.8682321040 \\
\hline 1.0 & 1.0 & 2.2033666610 & 2.2033666620 & 2.2471870730 & 2.2471870740 \\
\hline
\end{tabular}




\section{2D and 3D Graph Representation of Numerical Solution Fractional variable coefficients PDE}

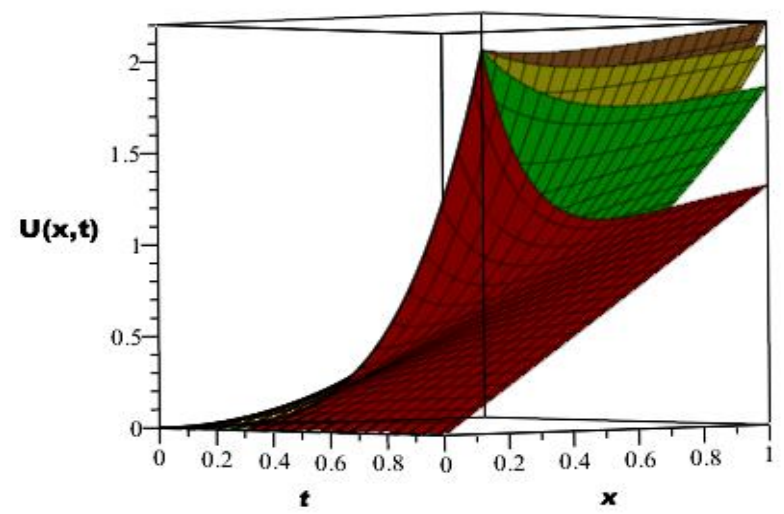

Fig. 1. Solution of fractional variable coefficients NIM, when $\lambda(x)=\frac{x}{1}, \frac{x}{3}, \frac{x}{5}, \frac{x}{7}$ Odd number 3D plot

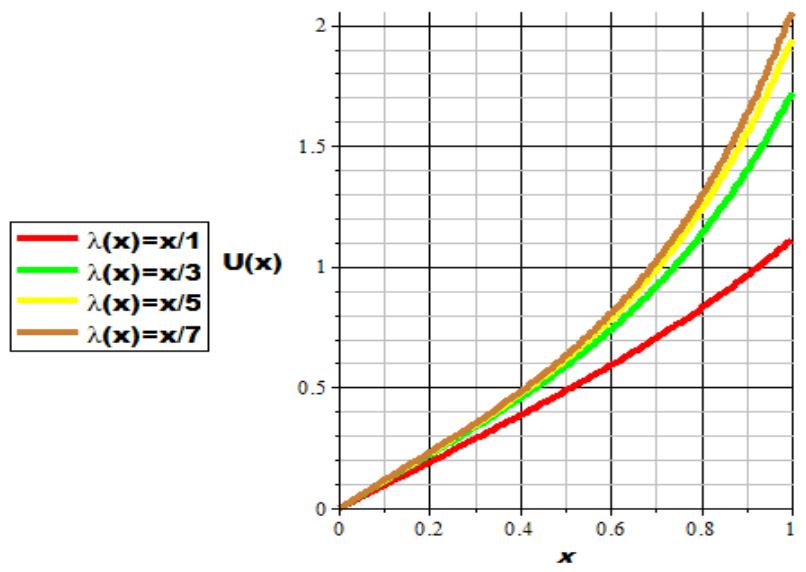

Fig. 2. Solution of fractional variable coefficients NIM, when $\lambda(x)=\frac{x}{1}, \frac{x}{3}, \frac{x}{5}, \frac{x}{7}$ at $t=0.5$ 2D plot

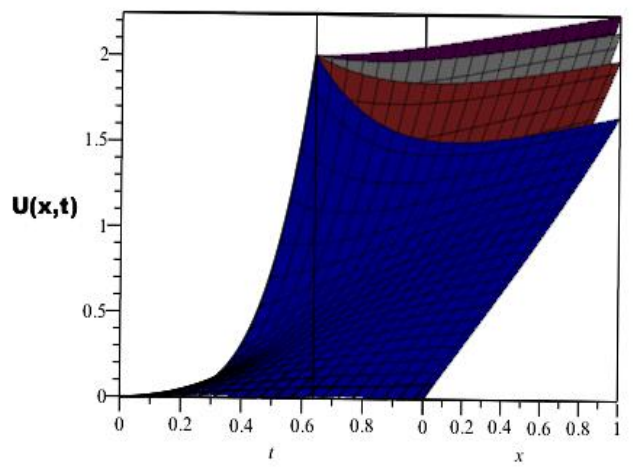

Fig. 3. Solution of fractional variable coefficients NIM, when $\lambda(x)=\frac{x}{2}, \frac{x}{4}, \frac{x}{6}, \frac{x}{8}$ Eveb number 3D plot 


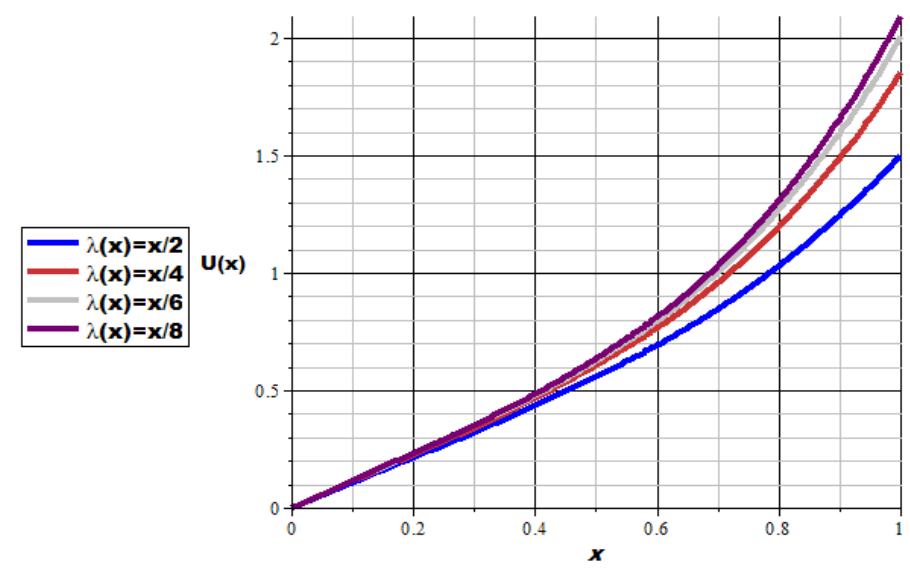

Fig. 4. Solution of fractional variable coefficients NIM, when $\lambda(x)=\frac{x}{2}, \frac{x}{4}, \frac{x}{6}, \frac{x}{8}$ at $t=0.52 \mathrm{D}$ plot

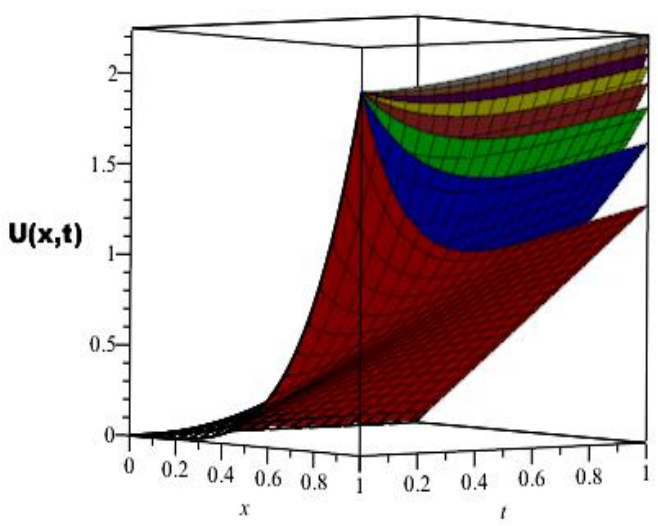

Fig. 5. Solution of fractional variable coefficients NIM, when $\lambda(x)=\frac{x}{1}, \frac{x}{2}, \frac{x}{3}, \ldots \frac{x}{8} 3 \mathrm{D}$ plot

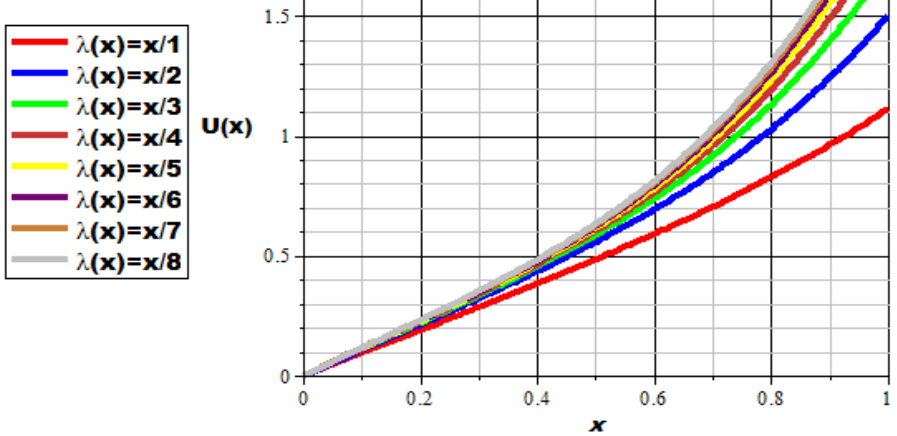

Fig.6. Solution of fractional variable coefficients NIM, when $\lambda(x)=\frac{x}{1}, \frac{x}{2}, \frac{x}{3}, \ldots \frac{x}{8}$ at $t=0.5$ 2D plot 


\section{Discussion and Conclusion}

\section{Discussion}

Fig.1 and Fig.2 illustrate the influence and trend of variable coefficient $\lambda(x)$ on equation (1) which indicate the numerical behaviours of integer $k$ when is odd numbers. As integer $k$ increasing tends to higher numerical solutions of $u(x, t)$. Similarly, Fig. 3 and Fig.4 show a similar trend and behaviours when integer $k$ is even numbers. Fig. 5 and Fig. 6 depict a combined plots of numerical trend of fractional variable coefficient when $k=1,2,3,4,5,6,7,8 \ldots$. . We finally established that as $k$ increases, the numerical solution of equation (1) is also increases but with little or no significant difference as $\lambda(x) \rightarrow \epsilon$.

\section{Conclusions}

In this paper, the nonhomogeneous fractional variable coefficient of partial differential equations are numerically investigated by a proposed MAPLE 18 algorithm written using New Iterative Method, which overcome the length computation and evaluation of integral involve. This approach is combines the following three advantages: easier, faster and reliable. A numerical example is provided in this paper and the distributions of solutions and absolute errors are shown to validate the accuracy of the proposed algorithm. Therefore, the simplicity and the accuracy of the proposed numerical scheme are verified. In view of this, we recommend the proposed new iterative Algorithm for general purpose in all areas of applied sciences and engineering especially in heat transfer, thermodynamics, population models, chemical kinetics and fluid mechanics

\section{References}

[1] H. Bulut, H. Mehmet Baskonus, and Seyma Tuluce the Solutions of Partial Differential Equations with Variable Coefficient by Sumudu Transform Method American Institute of Physics. Conference proceeding pp. 2-5: http://dx.doi.org/10.1063/1.4765475. 2012.

[2] E. L.Ortız, and H.Samara Numerical solution of Partial Differential equations with variable coefficients with an Operational approach to the tau method Comp \& Maths with Appls Vol. 10, pp. 5-13. 1984.

[3] J. Biazar and H. Ghazvini, Homotopy perturbation method for solving hyperbolic partial differential equations,Comput. Math. with Appl., vol. 56, no. 2, pp. 453-458, 2008.

[4] M.J. Jang, C.L. Chen, Y.C. Liu. Two-dimensional differential transform for partial differential equations. Applied Mathematics and Computation, 121 :261-270 2001.

[5] L. Shijun. On the homotopy analysis method for nonlinear problems. Applied Mathematics and Computation, 147 :499-513. 2004.

[6] G. Adomian. Solving frontier problems of physics: The decomposition method. Academic Publishers, Boston and London. 1994.

[7] J.H. He. A new approach to nonlinear partial differential equations. Commun Nonlinear Sci Numer Simul., 2 :2302351997.

[8] J.H. He. Homotopy perturbation technique. Computational, Methods in Applied Mechanics and Engineering., $178: 257-2621999$.

[9] M. Khan. An effective modification of the Laplace decomposition method for nonlinear equations. International Journal of Nonlinear Sciences and Numerical Simulation, 10 :1373-1376 2009.

[10] D. Kumar, S. Jagdev, R. Sushila Sumudu Decomposition Method for Nonlinear Equations. In International Mathematical Forum, 7 :515-521 2012.

[11] A. B Ratsos, M. E Hrhardt and I. T H. Famelis, A discrete Adomian decomposition method for discrete nonlinear Schrodinger equations, Appl. Math. Comput., 197, pp. 190-205 2008.

[12] M. Yaseen and M. Samraiz.The Modified New Iterative Method for Solving Linear and Nonlinear Klein-Gordon Equations New Iterative Method [ 2 ],vol. 6, no. 60, pp. 2979-2987 2018.

[13] R. Behl, A. Cordero, and J. R. Torregrosa, (2018) New Iterative Methods for Solving Nonlinear Problems with One and Several Unknowns, pp. 1-17, 2018.

[14] A. Mathematics, S. Bhalekar, and V. Gejji,New Iterative Method: Application to Partial Differential Equations," no. September 2008.

[15] C. Chun, A new iterative method for solving nonlinear equations, vol. 178, pp. 415-422. 200. 


\section{Authors' Profiles}

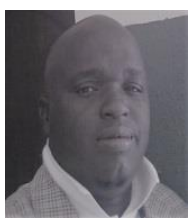

FALADE Kazeem Iyanda Ph.D is currently a Senior Lecturer in the Department of Mathematics Kano University of Science and Technology, Wudil Kano State Nigeria. His research areas are Numerical and Computational Mathematics. He is a member of Nigerian Mathematical Society (NMS), Mathematical Association of Nigeria (MAN), Nigerian Association of Mathematical Physics (NAMP), Nigerian Institutional of Professional Engineers and Scientist (NIPES) and Nigerian Society of Physical Sciences.

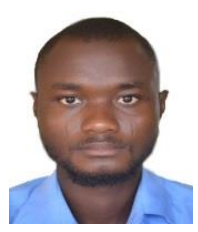

TIAMIYU Abd'gafar Tunde is currently a Master of Technology (M.Tech.) student in the Department of Mathematics, Federal University of Technology Minna, Nigeria. His research areas are in Computational and Applied Mathematics.

How to cite this paper: Falade K. I, Tiamiyu A.T," Numerical Solution of Partial Differential Equations with Fractional Variable Coefficients Using New Iterative Method (NIM)", International Journal of Mathematical Sciences and Computing(IJMSC), Vol.6, No.3, pp.12-21, 2020. DOI:10.5815/ijmsc.2020.03.02 